Mathematical Research Letters 1, 95-98 (1994)

\title{
ON THE DIAMETER OF PLANE ALGEBRAIC CURVES
}

\author{
Fedor A. Bogomolov
}

\section{Introduction}

The problem of a diameter of a plane complex algebraic curve was initially proposed by S. Frenkel. He conjectured that the diameter of a complex algebraic curve in the projective plane, $P^{2}$, is bounded in the induced metric independently of the degree of the curve. We assume that the metric on the projective plane is a standard Fubini-Study metric with the diameter of each projective line equal to 1. For example, the diameter, i.e. maximal distance in the induced metric, on any union of projective lines never exceeds 2. On the other hand M. Gromov conjectured that in fact diameters of complex algebraic curves in $P^{2}$ are unbounded unless there are some restrictions on the curves considered.

This article contains a proof of Gromov's conjecture. I describe further a procedure which enables one to construct, starting with any smooth curve, a new plane smooth curve with a diameter which approximately doubles the diameter of the initial curve. Hence by repeating this procedure we can produce smooth plane curves with a diameter exceeding any positive number. It gives a negative answer to the question of S. Frenkel and thus proves the Gromov conjecture. It is interesting to remark that the diameter is nevertheless bounded independently of the degree on a rather big class of plane curves.

$\S 1$

Let $C$ be a compact smooth algebraic curve in the complex projective plane $P^{2}$. We assume that Fubini-Study metric $h$ is given on $P^{2}$ and $h$ induces metric on $C$.

Definition. By a diameter $\mathrm{D}(\mathrm{C})$ of the curve $C$ in $P^{2}$ we shall denote the maximal distance between points of $C$ in the induced metric.

We shall assume that the diameter of any projective complex line is equal to 1 . Since $C$ is compact this maximum is achieved for some pair of points, say $x, y$ in $C$. We shall fix this pair of points in $C$.

Received September 19, 1993. 
Theorem 1.1. For any smooth curve $C$ and $\epsilon>0$ we can find another smooth curve $C^{\prime}$ such that the diameter $D\left(C^{\prime}\right)>2 D(C)-\epsilon$.

Proof of Theorem 1.1. We shall construct the curve $C^{\prime}$ in a small analytical neighborhood of $C$. In fact $C^{\prime}$ will be an infinitesimal deformation of $n C$ for $n$ big enough. It enables us to make all computations not in $P^{2}$, but in a normal bundle to the curve $C$. The normal bundle to $C$ has a natural metric which is equivalent to the metric in $P^{2}$ along $C$ up to the second order. Let us denote this normal bundle by $L$. It is equivalent to the restriction of $O(C)$ on $C$, where $O(C)$ is a locally free sheaf on $P^{2}$ defined by $C$ (see [2]).

If $C$ is of degree $m$ then the curves of multiple degree $m n$ which lie close to $C$ can be described as deformations of $n C$.

A natural map of the space of sections $H^{0}\left(P^{2}, O(m n)\right) \rightarrow H^{0}(n C, O(n m))$ is surjective with a one dimensional kernel. The corresponding projection onto the reduced curve $C$ defines an element of

$$
H^{0}(C, O(n m))=H^{0}(C, n L) .
$$

If we consider a decomposition of a section $s \in H^{0}\left(P^{2}, O(m n)\right)$ over the normal parameter to $C$ then the image of $s$ in $H^{0}(C, O(n m))$ coincides with the first order deformation of $n C$. The latter can be identifed also with a multisection of the normal line bundle $L$ to $C$, namely $n$-section. This multisection is induced from the section $s$ of $n L$ by a fiberwise map $n: L \rightarrow n L$. This map coincides with $z \rightarrow z^{n}$ on each fiber.

We have to choose $n$ and a section $s$ of $n L$ in order to double the diameter of $C$ on $S$, the preimage $n^{-1} s$. We need the following technical lemma.

Lemma 1.2. For any neighborhood $U$ of a point $x$ in $C$ we can find an integer $r$ such that there exists a section $s_{n} \in H^{0}(C, n L)$ for any $n \geq r$ with all zeroes contained in $U$.

Proof. Let us show that linear combinations of more than $r$ points in $U$ represent any divisor class of degree $r$ for $r$ big enough. Indeed all divisors of fixed degree are parametrised by the Jacobian, which is a compact torus.

Any small neighbourhood $U$ in $C$ generates the Jacobian. In particular if $g$ is the genus of $C$ then we can estimate the number $k$ from above as $g \times D(C) \epsilon^{-1}$. Here $D(C)$ is the diameter of $C$ and $\epsilon$ is the radius of the neighborhood $U$. The real dimension of the Jacobian is equal to $2 g$.

Therefore any class of divisors of degree more than $r$ has an effective representative with a support in $U$. In other words any bundle of degree more than $r$ has a section concentrated in $U$. 
Remark. If $s$ is a section of $n L$ over $C$ then $s^{k}$ is a section of $n k L$. The section $\tilde{s}=s^{k}+\nu s^{\prime}$ will have all of its zeroes inside the same neighbourhood if $s^{\prime}$ is any section in $H^{0}(C, n k L)$ and $\nu$ is small enough.

End of proof of Theorem 1.1. Let $s$ be a section satisfying the conditions of Lemma 1.2. Then the curve $S=n^{-1} s$ in a line bundle $L$ is a nonramified covering of $C$ outside a small neighbourhood $U$ of $x$. I recall that by assumption on $x$ there exists a point $y$ in $C$ such that the distance $d(x, y)=$ $D(C)$.

Let us take all the points $y_{i}$ which are the preimages of $y$ in $C$. Consider the distances between points $y_{i}$ in $S$. If the minimum is achieved on the line which intersect the preimage of $U$ in $S$ then the distance is more than $2 D(C)-\epsilon$ and hence $D(S)$ almost doubles the diameter of $C$. Suppose that this does not occur and $d\left(y_{i}, y_{j}\right)$ is equal to the length of some line connecting them outside of the preimage of $U$. The projection of this line in $C$ is closed and represents a nontrivial homology class.

The covering is defined over $C-U$ by integrating some one-dimensional closed form $\omega$. Assume that the pointwise norm of $\omega$ with respect to the above metric $h$ does not exceed some constant $M$ in $C-U$. If we take any $k$ and a small variation $\tilde{s}$ of $s^{k}$ as in the remark above then the resulting covering in the complimentary will correspond to the $k$-multiple of the cohomology class defining the covering for $s$.

Now we can choose appropriately the degree $N=n k$ of the cyclic ramification of $C-U$ by taking big enough $k$ and $\tilde{s}$ described above. The maximal distance between different preimages $y_{i}, y_{j}$ of $y$ along the lines in the preimage of $C-U$ will be estimated from below by $N M^{-1}$. Le $S$ be the curve obtained as the preimage $n^{-1} s$; then $S$ is ramified at zeroes of the section $s$. The curve $S$ lies in a normal line bundle $L$ and not in the neighborhood of $C$ in $P^{2}$. We can find a curve $C^{\prime}$ close to $n C$ which is smooth and such that $S$ approximates $C^{\prime}$ up to second order. Since both curves $S$ and $C^{\prime}$ are smooth, the induced metric on $C^{\prime}$ is close to the metric induced on $S$ from the normal bundle. In particular if we take $N$ greater than $2 D(C) \times M$ then the diameter of $S$ will be greater than $2 D(C)-\epsilon$. Hence we can find a curve $C^{\prime}$ which is infinitesimally close to $C$ and such that $D\left(C^{\prime}\right)$ is greater than $2 D(C)-\epsilon$. This proves Theorem 1.1.

The above procedure can be repeated for a newly constructed curve $C^{\prime}$ and so on. It proves therefore the following general fact:

Corollary 1.3. Diameters of complex curves in the projective plane are not bounded from above by any constant.

Since the set of curves of fixed degree is compact diameters are bounded on the set of curves of bounded degree. It would be natural then to consider 
nontrivial upper bounds for the maxim al diameter of the curves of fixed degree. Presumably there exists an estimate from above which is logarithmic on the degree of a curve. Nevertheless, we hypothesize that the curves in some special classes have bounded diameter.

Conjecture. The hyperelliptic curves have a diameter bounded from above by 4 being considered with standard imbedding in $P^{2}$.

Similar estimates may be true also for other classes of curves with bounded degree of some projection on $P^{1}$ induced from a projection of the plane.

Remark. By the action of the projective group any curve can be deformed into a small neighbourhood of a family of lines with a common intersection point. The metric on such a curve has a diameter close to 2 . Thus all spectra of the variation of a diameter can be achieved on projectively equivalent curves if we restrict ourselves to the curves of bounded degree.

\section{Acknowledgement}

I am grateful to M. Gromov for introducing me to the problem and for helpful discussions. The solution was obtained during my stay at the University of Maryland and the final version was written during my visit at SFB-170 of the Goettingen University. I am thankful to both of these institutions for support and excellent working conditions.

\section{References}

1. Arthur L. Besse, Einstein manifolds, Springer-Verlag, 1987.

2. W. Barth, C. Peters and A. Van de Ven, Compact complex surfaces, Springer-Verlag, 1987.

Steklov Institute of M athematics, M oscow

Current address: Department of Mathematics, University of Maryland, College Park, MD 20742

E-mail address: bogomol@charlotte.umd.edu 